\title{
Fabry-Perot Vapor Microsensors Fabricated onto Fibre Endface by Multiphoton Polymerization Technique
}

\author{
Vasileia Melissinaki ${ }^{1,2}$, Maria Vamvakaki ${ }^{1,3}$, Maria Farsari ${ }^{1}$, Stavros Pissadakis ${ }^{1}$ \\ 1. Foundation for Research and Technology-Hellas (FORTH), Institute of Electronic Structure and Laser (IESL), 71110 Heraklion, Greece \\ 2. Department of Physics, University of Crete, Heraklion, Greece \\ 3. Department of Materials Science and Technology, University of Crete, Heraklion, Greece
}

We report on the fabrication of two different Fabry-Perot optical resonators on the endface of a SMF28 fibre, by employing Direct Laser Writing by multi-photon polymerization [1, 2]. We explore these endface fibre sensing probes for tracing common organic solvents [3]. The devices developed have been optimised for operation at the spectral region lying between $1415 \mathrm{~nm}$ to1655nm, while being interrogated in reflection mode using a simple 50/50 fibre coupler.

The laser used was a Ti:sapphire unit emitting at $800 \mathrm{~nm}, 200 \mathrm{fs}$ pulses at a repetition rate of $50-80 \mathrm{MHz}$. The beam projection system was based on an inverse microscope; beam steering was performed using a $\mathrm{x}-\mathrm{y}$ galvanometric mirror scanning the beam through a high magnification objective. Lateral resolution was of the order of 250nm [1]. The material that used for the fabrication of the endface microstructures is a zirconium-silicon, organic-inorganic hybrid photosensitive material [4]. The first microstructure consists of a thin, flat membrane suspended on four pillars attached onto the silica fibre endface (Fig. 1a). This architecture allows the formation of a small air cavity between the end face of the fibre and the suspended thin membrane, which acts as a multilayer Fabry-Perot resonator, providing interrogation capabilities of the media inside the air cavity section; or attached on the membrane endface [5]. Liquid or gaseous media can be trapped between the empty gap of the microdrum resonator, detecting refractive index or absorption changes in reflection mode. The specific cavity demonstrated herein consists of a 14.4um air gap formed between the fibre endface and the photopolymerised structure, while the membrane thickness is $10.4 \mathrm{um}$, resulting in the periodic modulation of the reflected optical spectrum by notches of $\sim 29 \mathrm{~dB}$ in amplitude strength with a free spectral range of $\sim 87 \mathrm{~nm}$.

(a)

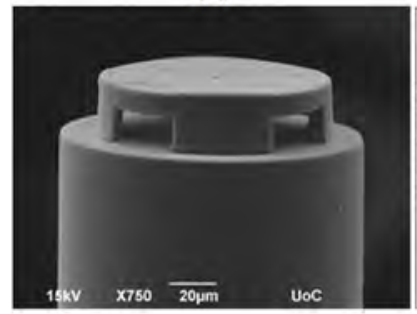

(b)

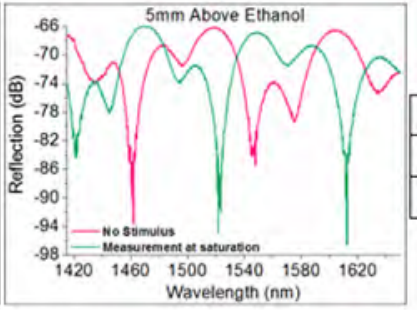

(c)

Fig 1: (a) SEM image of the microdrum resonator on the endface of the fibre, (b) Reflection spectrums of ethanol, (c) Shifts of the Fabry-Perot for ethanol and 1-propanol, for two different distances of the sensing head above solvents.

Initially, we have tested the operation of this sensing probe for measuring vapours of two different alcohols, ethanol and 1-propanol (Fig. 1b), in an open air setup. The sensing head was placed above the solvent's surface resting inside a small glass tube and measurements were taken for those alcohols, at two different distances, $5 \mathrm{~mm}$ and $1 \mathrm{~mm}$ above the liquid surface. As shown above, for $5 \mathrm{~mm}$ above ethanol, the shift of the Fabry-Perot reaches the value 62.27nm, after 1 hour of exposure, that the system was saturated (Fig. 1c), while for 1mm distance the corresponding shift is $14.79 \mathrm{~nm}$. We observe that the changes are more significant for solvents with higher vapor pressure, than those for lower vapor pressure, where the sensor is more stable. Moreover, system's stability improves upon decreasing the distance between sensor's head and the surface of the solvent. The sensing head is fully recovered in terms of spectral response to the initial state, after removing from the alcohol stimulus.

Then, for avoiding the multiple Fabry-Perot resonation between the two endfaces of the thin membrane, which in turn complicates the interrogation spectrum, a $\sim 20^{\circ}$ funnel was created homocentrically with the membrane (Fig.2a).

(a)

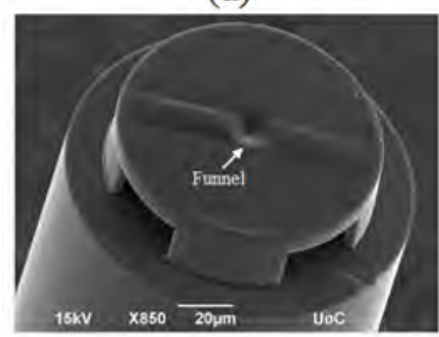

(b)

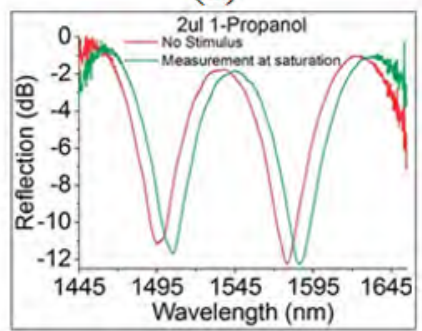

(c)

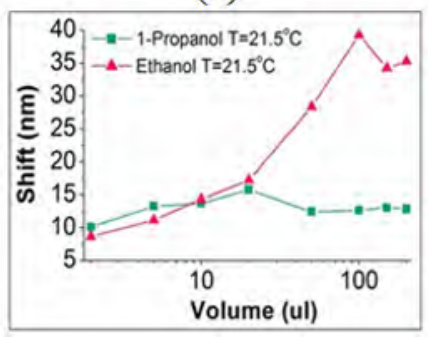

Fig 2: (a) SEM image of the microstructure on the endface of the fibre, (b) Reflection spectrums of 2ul 1-Propanol, (c) Shifts of the Fabry-Perot for two different alcohols for a variety of volumes. 
The funnel walls inclination directed the reflection from the outer endface of the membrane away from the cavity area. The specific cavity demonstrated herein consists of a 16.9um air gap formed between the fibre endface and the photopolymerised structure, resulting in the periodic modulation of the reflected optical spectrum by notches of $\sim 10 \mathrm{~dB}$ in amplitude strength with a free spectral range of $\sim 80 \mathrm{~nm}$.

We tested again the operation of this sensing probe, for measuring vapours of two different alcohols those of 1propanol and ethanol, at several different gaseous concentrations spanning from 11mbar to 70mbar, injected inside a $500 \mathrm{ml}$ volume steel cell this time. As shown above, for $2 \mathrm{ul}$ of 1-Propanol the shift of the Fabry-Perot was $10.08 \mathrm{~nm}$ for a saturated pressure of $11 \mathrm{mbar}$ (Fig. 1b), while for the same volume of Ethanol the shift was $8.61 \mathrm{~nm}$ for $12.5 \mathrm{mbar}$ maximum pressure in the cell (Fig.1c). We believe that the fundamental mechanism for the vapour sensing is specie adsorption since calibration experiments using inert gasses resulted in substantially smaller wavelength shifts of the Fabry-Perot notches. The sensing head is fully recovered as well, after exposure to ambient air or nitrogen.

Further work is carried out for fully characterizing and optimizing this novel sensing probe and exploiting its operational characteristics for penetrating into selective, bio and chemo-sensing.

\section{References}

[1] M. Farsari, M. Vamvakaki and B. N. Chichkov, "Multiphoton polymerization of hybrid materials", J. Opt. 12, 124001 (2010).

[2] M. Malinauskas, A. Zukauskas, V. Purlys, A. Gaidukeviciute, Z. Balevicius, A. Piskarskas, C. Fotakis, S. Pissadakis, D. Gray, R. Gadonas, M. Vamvakaki and M. Farsari, "3D microoptical elements formed in a photostructurable germanium silicate by direct laser writing", Opt. Lasers Eng. 50, 1785 (2012).

[3] M. Konstantaki, A. Klini, D. Anglos, and S. Pissadakis, "An ethanol vapor detection probe based on a ZnO nanorod coated optical fiber long period grating", Opt. Exp. 20, 8472 (2012).

[4] I. Sakellari, E. Kabouraki, D. Gray, V. Purlys, C. Fotakis, A. Pikulin, N. Bityurin, M. Vamvakaki and M. Farsari, "Diffusion-Assisted HighResolution Direct Femtosecond Laser Writing", ACS Nano 6, 2302 (2012).

[5] S. Pevec and D. Donlagic, "Miniature all-fiber Fabry-Perot sensor for simultaneous measurement of pressure and temperature", Appl. Opt. 51, 4536 (2012). 